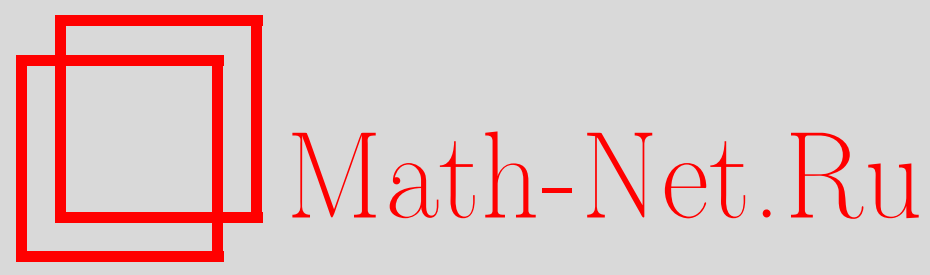

Б. Д. Гельман, Точки покоя обобщенных динамических систем, Матем. заметки, 1999, том 65, выпуск 1, 28-36

DOI: https://doi.org/10.4213/mzm1025

Использование Общероссийского математического портала Math-Net.Ru подразумевает, что вы прочитали и согласны с пользовательским соглашением http://www. mathnet.ru/rus/agreement

Параметры загрузки:

IP: 54.224 .135 .184

26 апреля 2023 г., 09:38:04

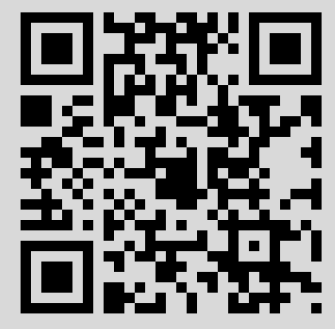




\title{
ТОЧКИ ПОКОЯ ОБОБЩЕННЫХ ДИНАМИЧЕСКИХ СИСТЕМ
}

\author{
Б. Д. Гельман
}

\begin{abstract}
В настоящей статье выделяется класс обобщенных динамических систем, у которых интегральная воронка (множество траекторий системы, выходящих из данной точки) является ацикличным множеством в пространствах кривых. В этом классе динамических систем для доказательства существования точек покоя удается применить теорию неподвижных точек многозначных отображений. На этом пути удается получить новые теоремы о точках покоя обобщенных динамических систем.
\end{abstract}

Библиографоия: 11 названий.

Обобшенные динамические системы возникли в связи с изучением обыкновенных дифференциальных уравнений без единственности и дифференциальных включений. Основы этой теории были заложены в работах А. Д. Мьшкиса, Е. А. Барбашина, Б. М. Будака, Е. Роксина и др.

В работе Мышкиса [1] была поставлена задача исследования точек покоя обобщенных динамических систем. В ней данная задача сводилась к изучению неподвижных точек многозначных отображений, образы которых имели сложную топологическую структуру. Однако, эта задача оказалась очень сложной, так как описать структуру образов многозначного отображения (возникающего при изучении этой задачи в размерностях больше 1) оказалось невозможно. Некоторые результаты о существовании точек покоя обобщенных динамических систем были получены также в работах [2], [3].

В настоящей статье выделяется класс обобщенных динамических систем, для которых соответствуюшие многозначные отображения имеют специальный вид: они представляются в виде композиции однозначного непрерьвного отображения и многозначного отображения с ациклическими образами. Так как для таких отображений развита теория неподвижных точек (см., например, [2], [4]), удается доказать некоторые теоремы о существовании точек покоя для динамических систем из этого класса.

Необходимые в дальнейшем сведения из теории обобшенных динамических систем содержатся в монографиях [5]-[7].

1. Основные факты теории обобщенных динамических систем. Пусть $Y-$ подмножество банахова пространства $E$. Обозначим через $\mathrm{K}(Y)$ множество всех непустых компактных подмножеств в $Y$, через $\mathrm{Kv}(Y)$ множество всех непустых компактных выпуклых подмножеств в $Y$.

Работа выполнена при частичной поддержке Российского фонда фундаментальных исследований, грант № 96-01-00360. 
Многозначное отображение (М-отображение) метрического пространства $X$ в метрическое пространство $Z$ - это соответствие, сопоставляюшее каждой точке $x \in X$ непустое подмножество $F(x) \subset Z$, назьваемое образом точки $x$. В дальнейшем, если образы М-отображения $F$ являются компактами, то будем записьвать это следуюшим образом: $F: X \rightarrow \mathrm{K}(Z)$. Аналогично, $F: X \rightarrow \mathrm{Kv}(Z)$ означает, что образы $F(x)$ являются вьпуклыми компактными множествами.

Графиком многозначного отображения $F: X \rightarrow \mathrm{K}(Z)$ назьвается множество

$$
\Gamma_{X}(F)=\{(x, z) \mid z \in F(x), x \in X\} \subset X \times Z
$$

Естественно определены проекции $t: \Gamma_{X}(F) \rightarrow X, t(x, z)=x$, и $r: \Gamma_{X}(F) \rightarrow Z$, $r(x, z)=z$.

Очевидно, что для любого $x \in X$ справедливо равенство $F(x)=r \cdot t^{-1}(x)$.

Всюду в дальнейшем М-отображения обозначаются прописными, а однозначные строчными буквами.

ОПРЕДЕЛЕНИЕ 1. Многозначное отображение $F: X \rightarrow C(Z)$ назьвается полунепрерывным сверху в точке $x_{\circ} \in X$, если для любого открытого множества $V \subset Z$, $V \supset F\left(x_{\circ}\right)$, сушествует открытая окрестность $U$ точки $x_{\circ}$ такая, что $F(U) \subset V$.

Если отображение $F$ полунепрерывно сверху в каждой точке $x \in X$, то оно назьвается полунепрерывным сверху.

ОПРЕДЕЛЕНИЕ 2. Многозначное отображение $F: X \rightarrow C(Z)$ называется полунепрерывным снизу в точке $x_{\circ} \in X$, если для любого открытого множества $V \subset Z$ такого, что $F\left(x_{\circ}\right) \cap V \neq \varphi$, найдется открытая окрестность $U$ точки $x_{\circ}$ такая, что $F(x) \cap V \neq \varphi$ для любого $x \in U$.

Если $F$ полунепрерьвно снизу в каждой точке $x \in X$, то оно называется полунепрерывным снизу.

ОПРЕДЕЛЕНИЕ 3. Многозначное отображение $F: X \rightarrow C(Z)$ назьвается непрерывны.м, если оно одновременно полунепрерывно сверху и снизу.

Пусть $E$-банахово пространство, $X \subset E, F: X \rightarrow C(E)$-некоторое М-отображение. Точку $x_{\circ}$ будем называть неподвижной точкой М-отображения $F$, если $x_{\circ} \in F\left(x_{\circ}\right)$.

Существует несколько различных определений обобщенной динамической системы (динамической системы без единственности). Наиболее распространенным является определение, предложенное Барбашиным [8].

Пусть $X$ - топологическое пространство, $\mathbb{R}$ - множество вещественных чисел, $\mathbb{R}_{+}-$ множество неотрицательных чисел.

ОПРЕДЕЛЕНИЕ 4. Многозначное отображение $\Psi: X \times \mathbb{R} \rightarrow \mathrm{K}(X)$ называется обобщенной динамической системой, если вьполнены следуюшие условия:

1) $\Psi(\cdot, 0): X \rightarrow X$ является id $_{X}$;

2) для любых $x \in X$ и $t_{1}, t_{2} \in \mathbb{R}, t_{1} \cdot t_{2} \geqslant 0$, имеем $\Psi\left(\Psi\left(x, t_{1}\right), t_{2}\right)=\Psi\left(x, t_{1}+t_{2}\right)$;

$3)$ для любых $x \in X, t \in \mathbb{R}$ из того, что $y \in \Psi(x, t)$ следует, что $x \in \Psi(y,-t)$;

4) М-отображение $\Psi$ полунепрерьвно сверху;

$5)$ для любого $x_{0} \in X$ М-отображение $\Psi\left(x_{0}, \cdot\right): \mathbb{R} \rightarrow K(X)$ непрерывно. 
Примером обобшенной динамической системы является система, задаваемая оператором сдвига по траекториям автономного дифференциального включения (или дифференциального уравнения без единственности): $\dot{x} \in F(x)$, где $F: X \rightarrow \mathrm{Kv}\left(\mathbb{R}^{n}\right)$ полунепрерьвное сверху М-отображение и решения данного включения нелокально продолжимы на всю числовую ось.

ОПРЕДЕЛЕНИЕ 5. Траекторией обобщенной динамической системы на отрезке $[a, b] \subset \mathbb{R}$ назьвается непрерьвное отображение $x:[a, b] \rightarrow X$, удовлетворяющее для любых $t_{0}, t_{1} \in[a, b]$ соотношению $x\left(t_{1}\right) \in \Psi\left(x\left(t_{0}\right), t_{1}-t_{0}\right)$.

В дальнейшем $X$ является метрическим пространством.

Справедливы следуюшие свойства траекторий обобщенных динамических систем (см., например, [5]).

Свойство 1. Всякая траектория обобщенной динамической системы является непрерывным отображением.

Свойство 2. Если $x_{1}$ - траектория обобщенной динамической системы на промежутке $[a, b]$, а $x_{2}$ - траектория этой системы на промежутке $[b, c]$, причем $x_{1}(b)=x_{2}(b)$, то отобрахсение $x:[a, c] \rightarrow X$,

$$
x(t)= \begin{cases}x_{1}(t), & \text { если } t \in[a, b], \\ x_{2}(t), & \text { если } t \in(b, c],\end{cases}
$$

также является траекторией этой системы на промежутке $[a, c]$.

Свойство 3. Если $\Psi$ - обобщенная динамическая система, то для любих $a \leqslant b$, $x_{1} \in \Psi\left(x_{0}, b-a\right)$ на отрезке $[a, b]$ существует траектория $x$ такая, что $x(a)=x_{0}$, $x(b)=x_{1}$.

Пусть $A \subset X$. Обозначим через $\Sigma(A,[a, b])$ множество траекторий $x$ системы $\Psi$ на отрезке $[a, b]$ таких, что $x(a) \in A$.

Свойство 4. Пусть $(X, \rho)$ - метрическое пространство, $\Psi: X \times \mathbb{R} \rightarrow \mathrm{K}(X)-$ обобщенная динамическая система. Тогда для любого компактного подмножества $A \subset X$ и любого $[a, b] \subset \mathbb{R}$ множество $\Sigma(A,[a, b])$ компактно в топологии равномерной сходимости.

Заменив в определении 4 числовую прямую $\mathbb{R}$ на $\mathbb{R}_{+}$и исключив условие 3 ), получим определение односторонней обобшенной динамической системы. Для односторонней динамической системы естественно определяется понятие траектории и справедливы свойства, аналогичные свойствам 1-4. Кроме этого, справедливо следующее свойстBO.

Лемма 1. Пусть $X$ - компактное метрическое пространство, $\Psi: X \times \mathbb{R}_{+} \rightarrow$ $\mathrm{K}(X)$ - односторонняя динамическая система. Тогда М-отображсение $\Sigma: X \rightarrow$ $\mathrm{K}\left(C_{([0, t], X)}\right), \Sigma(x)=\Sigma(x,[0, T])$, является полунепрерывным сверху.

Доказательство этой леммы очевидно.

2. Аппроксимируемые динамические системы. Пусть $\Psi: X \times \mathbb{R}_{+} \rightarrow \mathrm{K}(X)-$ односторонняя динамическая система. 
ОПРЕДЕЛЕНИЕ 6 . Будем говорить, что односторонняя динамическая система $\Psi$ является аппроксимируемой на множестве $X$, если существует последовательность динамических систем $\left\{f_{n}\right\}, f_{n}: X \times \mathbb{R}_{+} \rightarrow X$ (аппроксимирующее семейство) таких, что вьполнены следуюшие условия:

1) для любых $(x, t) \in X \times \mathbb{R}_{+}$предел

$$
\lim _{n \rightarrow \infty} \rho\left(f_{n}(x, t) ; \Psi(x, t)\right)=0 ;
$$

2) для любых $\varepsilon>0, T>0$ сушествует $\delta=\delta(\varepsilon, T)>0$ такое, что для $t_{0}, t_{1} \in[0, T]$, $\left|t_{0}-t_{1}\right|<\delta$, справедливо неравенство

$$
\rho\left(f_{n}\left(x, t_{0}\right) ; f_{n}\left(x, t_{1}\right)\right)<\varepsilon
$$

для любых $n$ и $x \in X$.

ПримеР. Пусть $F: \mathbb{R}^{n} \rightarrow \mathrm{Kv}\left(\mathbb{R}^{n}\right)$ - полунепрерывное сверху М-отображение. Пусть существует число $a$ такое, что

$$
\max _{y \in F(x)}\|y\| \leqslant a .
$$

Рассмотрим дифференциальное включение $\dot{x} \in F(x)$. При сделанных предположениях решения задачи Коши этого включения существуют и нелокально продолжимы на множество $\mathbb{R}_{+}$при любых начальных условиях.

Рассмотрим одностороннюю обобщенную динамическую систему $\Psi$, порожденную оператором сдвига по траекториям этого дифференциального включения. Нетрудно доказать, что эта динамическая система является аппроксимируемой в $\mathbb{R}^{n}$. Здесь, апшроксимирующее семейство порождено операторами сдвига по траекториям дифференциальных уравнений, правые части которых являются $\varepsilon$-апшроксимациями М-отображения $F$.

Изучим свойства интегральной воронки $\Sigma(x,[a, b])$ аппроксимируемой динамической системы. Нам понадобится следуюшее понятие.

Пусть $X$ - метрическое пространство, $H^{n}(X, G)$ - когомологии Александера-Чеха пространства $X$ с коэффициентами в групше $G$ (см., например, [9]).

ОПРЕДЕЛЕНИЕ 7. Будем говорить, что пространство $X$ является $G$-аuикличныл если приведенные когомологии $\bar{H}^{n}(X, G)=0$ для любого $n \geqslant 0$.

В дальнейшем будем опускать $G$ и говорить просто об ацикличности, считая группу $G$ фиксированной.

Ацикличность $X$ означает, что это множество устроено достаточно просто, в нем отсутствуют "дыры".

Лемма 2. Пусть $X$ - метрическое пространство, $A$ - компактное подмножество в $X$. Пусть существует последовательность полунепрерывных сверху М-отображсений $\left\{F_{n}\right\}, F_{n}:[0,1] \times A \rightarrow \mathrm{K}(X)$, удовлетворяюших условиям:

1) мнохество $F_{n}(\lambda, x)$ является ацикличным для любих $(\lambda, x) \in[0,1] \times A u$ любого $n$

2) для всякого $\varepsilon>0$ существует номер $n_{0}$ такой, что для любого $n \geqslant n_{0}$ мнозсество $F_{n}([0,1] \times A) \subset U_{\varepsilon}(A)$; 
3) для любых $x \in A, n \in N$ выполнено включение $x \in F_{n}(0, x)$;

4) для любого $n$ существует точка $a_{n} \in X$ такая, что $a_{n} \in F_{n}(1, x)$ для всех $x \in A$.

Тогда множество $A$ является ацикличным.

ДокАЗАТЕЛЬСТво. Пусть $\varepsilon-$ произвольное положительное число, $F_{n}([0,1] \times A) \subset$ $U_{\varepsilon}(A)$. Пусть отображения $i_{n}, j_{n}: A \rightarrow X$ определены соотношениями $i_{n}(x)=x$, $j_{n}(x)=a_{n}$. Обозначим через $I$ отрезок $[0,1]$. Тогда имеет место коммутативная диаграмма

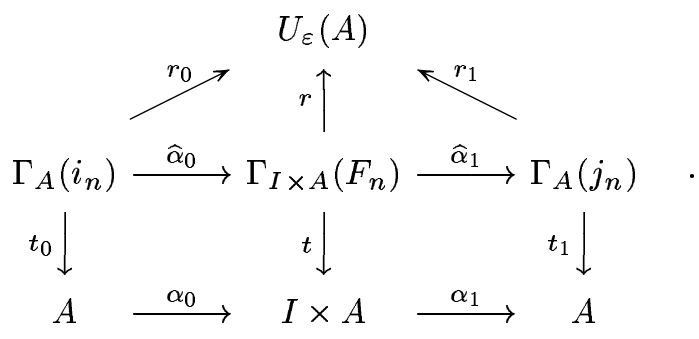

Здесь $\alpha_{0}(x)=(0, x), \alpha_{1}(x)=(1, x) ; t_{0}, t, t_{1}$ - проекции из графиков на области определений; $r_{0}, r, r_{1}-$ проекции из графиков на области значений; $\widehat{\alpha}_{0}$ и $\widehat{\alpha}_{1}-$ соответствующие вложения.

Переходя к индуцированной диаграмме в приведенных когомологиях, замечаем, что гомоморфизмы $t_{0}^{*}, t_{1}^{*}, \alpha_{0}^{*}, \alpha_{1}^{*}$ являются изоморфизмами, а $t^{*}$ - изоморфизм в силу теоремы Виеториса (см. [10]). Тогда гомоморфизмы $\widehat{\alpha}_{0}^{*}$ и $\widehat{\alpha}_{1}^{*}$ также являются изоморфизмами. Следовательно, $r_{0}^{*}=\widehat{\alpha}_{0}^{*} \cdot\left(\widehat{\alpha}_{1}^{*}\right)^{-1} \cdot r_{1}^{*}$. Так как гомоморфизм $r_{0}^{*}$ может быть представлен в виде $\widehat{r}_{1}^{*} \cdot i^{*}$, где

$$
\Gamma_{A}\left(j_{n}\right) \stackrel{\widehat{r}_{1}}{\rightarrow} a_{n} \stackrel{i}{\rightarrow} U_{\varepsilon}(A),
$$

то $r_{1}^{*}$ является нулевым гомоморфизмом. Следовательно, $r_{0}^{*}=0$.

Таким образом, вложение $i_{n}: A \rightarrow U_{\varepsilon}(A)$ индуцирует нулевой гомоморфизм для любого $\varepsilon>0$. Так как в силу жесткости теории когомологий Александера-Чеха (см., например, [9])

$$
\underset{\lim }{\longrightarrow} \bar{H}^{k}\left(U_{\varepsilon}(A), G\right)=\bar{H}^{k}(A, G),
$$

то $\bar{H}^{k}(A, G)=0$ для любого $k \geqslant 0$.

Лемма доказана.

ТЕОрема 1. Пусть $X$ - компактное метрическое пространство. Пусть $\Psi: X \times$ $\mathbb{R}_{+} \rightarrow \mathrm{K}(X)$ - односторонняя динамическая система. Если $\Psi$ является аппроксимируемой на $X$, то мнохество $\Sigma\left(x_{0},[0, T]\right) \subset C_{([0, T], X)}$ является $G$-ацикличным для любой группь $G$ и любых $x_{0} \in X u T>0$.

ДокАЗАтЕЛЬСтво. Пусть $f_{n}: X \times \mathbb{R}_{+} \rightarrow X-$ аппроксимируюшее семейство динамической системы $\Psi$. Рассмотрим последовательность непрерьвных отображений $\left\{\varphi_{n}\right\}, \varphi_{n}: \Sigma\left(x_{0},[0, t]\right) \times[0,1] \rightarrow C_{([0, T], X)}$, где $\varphi_{n}$ определено условием

$$
\varphi_{n}(u, \lambda)(t)= \begin{cases}u(t), & \text { если } t \in[0,(1-\lambda) T], \\ f_{n}(u((1-\lambda) T), t-(1-\lambda) T), & \text { если } t \in((1-\lambda) T, T] .\end{cases}
$$

Покажем, что последовательность $\left\{\varphi_{n}\right\}$ удовлетворяет условиям леммы 2. 
Действительно, образ $\varphi_{n}(u, \lambda)$ - это одна точка, т.е. $G$-ацикличное множество. При $\lambda=0$ получаем, что $\varphi_{n}(u(\cdot), 0)(t)=u(t)$, т.е. является единичным отображением. При $\lambda=1$ получаем, что $\varphi_{n}(u(\cdot), 1)=f_{n}\left(x_{0}, t\right)$, т.е. не зависит от выбора $u(\cdot) \in \Sigma\left(x_{0},[0, t]\right)$.

Осталось доказать, что для любого $\varepsilon>0$ существует номер $n_{0}$ такой, что для любого $n \geqslant n_{0}$ и любых $u(\cdot) \in \Sigma\left(x_{0},[0, T]\right), \lambda \in[0,1]$ выполнено включение

$$
\varphi_{n}(u(\cdot), \lambda) \in U_{\varepsilon}\left(\Sigma\left(x_{0},[0, T]\right)\right) \subset C_{([0, t], X)} .
$$

Предположим противное. Тогда существуют $x_{0}, \varepsilon_{0}>0$, последовательности $\left\{\lambda_{n}\right\} \in$ $[0,1],\left\{u_{n}(\cdot)\right\} \in \Sigma\left(x_{0},[0, T]\right)$ такие, что

$$
\rho\left(\varphi_{n}\left(u_{n}(\cdot), \lambda_{n}\right) ; \Sigma\left(x_{0},[0, T]\right)\right) \geqslant \varepsilon_{0} .
$$

Без ограничения обшности можно считать, что $\lambda_{n} \rightarrow \lambda_{*} \in[0,1], u_{n}(\cdot) \rightarrow u_{*} \in \Sigma\left(x_{0}\right.$, $[0, T])$.

Положим $x_{n}(\cdot)=\varphi_{n}\left(u_{n}(\cdot), \lambda_{n}\right)$. В силу сделанных предположений множество $A=$ $\left\{x_{n}(\cdot)\right\}_{n=1}^{\infty} \subset C_{([0, T], X)}$ является равностепенно непрерьвньм. Следовательно, из последовательности $\left\{x_{n}(\cdot)\right\}$ можно выделить сходящуюся подпоследовательность. Без ограничения обшности будем считать, что сама последовательность $\left\{x_{n}(\cdot)\right\}$ сходится к $x_{*}(\cdot)$ в пространстве $C_{([0, T], X)}$. Покажем, что $x_{*}(\cdot)$ принадлежит множеству $\Sigma\left(x_{0}\right.$, $[0, T])$.

Так как $x_{n}(t)=u_{n}(t)$ при $t \in\left[0,\left(1-\lambda_{n}\right) T\right]$, то $x_{*}(t)=u_{*}(t)$ при $t \in\left[0,\left(1-\lambda_{*}\right) T\right]$, т.е. $x_{*}(\cdot)$ является траекторией динамической системы $\Psi$ на промежутке $\left[0,\left(1-\lambda_{*}\right) T\right]$, выходящей из точки $x_{0}$.

Покажем теперь, что $x_{*}(\cdot)$ является траекторией динамической системы $\Psi$ на промежутке $\left[\left(1-\lambda_{*}\right) T, T\right]$. Пусть $t_{0}, t_{1} \in\left[\left(1-\lambda_{*}\right) T, T\right], t_{0}<t_{1}$. Тогда для любого $\varepsilon \in\left(0, t_{1}-t_{0}\right)$ существует номер $n_{0}$ такой, что $\left(1-\lambda_{n}\right) T<t_{0}+\varepsilon<t_{1}$ при $n \geqslant n_{0}$.

По построению функций $x_{n}(\cdot)$ имеем

$$
\begin{aligned}
x_{n}\left(t_{1}\right) & =f_{n}\left(u_{n}\left(\left(1-\lambda_{n}\right) T\right) ; t_{1}-\left(1-\lambda_{n}\right) T\right) \\
& =f_{n}\left(f_{n}\left(u_{n}\left(\left(1-\lambda_{n}\right) T\right) ; t_{0}+\varepsilon-\left(1-\lambda_{n}\right) T\right), t_{1}-t_{0}-\varepsilon\right) .
\end{aligned}
$$

Заметим, что

$$
f_{n}\left(u_{n}\left(\left(1-\lambda_{n}\right) T\right) ; t_{0}+\varepsilon-\left(1-\lambda_{n}\right) T\right)=x_{n}\left(t_{0}+\varepsilon\right) .
$$

Тогда

$$
x_{n}\left(t_{1}\right)=f_{n}\left(x_{n}\left(t_{0}+\varepsilon\right), t_{1}-t_{0}-\varepsilon\right) .
$$

В силу определения 6

$$
\lim _{n \rightarrow \infty} \rho\left(f_{n}\left(x_{n}\left(t_{0}+\varepsilon\right), t_{1}-t_{0}-\varepsilon\right) ; \Psi\left(x_{n}\left(t_{0}+\varepsilon\right), t_{1}-t_{0}-\varepsilon\right)\right)=0 .
$$

Откуда в силу полунепрерывности сверху М-отображения $\Psi$, равностепенной непрерьвности последовательности $\left\{f_{n}\right\}$ и сходимости $\left\{x_{n}(\cdot)\right\} \rightarrow x_{*}(\cdot)$ получаем включение

$$
x_{*}\left(t_{1}\right) \in U_{\eta}\left(\Psi\left(x_{*}\left(t_{0}\right), t_{1}-t_{0}\right)\right)
$$

для любого $\eta>0$. Следовательно, $x_{*}\left(t_{1}\right) \in \Psi\left(x_{*}\left(t_{0}\right), t_{1}-t_{0}\right)$, т.е. $x_{*}(\cdot)$ является траекторией динамической системы $\Psi$ на промежутке $\left[\left(1-\lambda_{*}\right) T, T\right]$. Из свойства 2 получаем, что $x_{*}(\cdot) \in \Sigma\left(x_{0},[0, T]\right)$. Полученное противоречие и доказьвает теорему. 
3. Точки покоя обобщенных динамических систем. Пусть $\Psi: X \times \mathbb{R}_{+} \rightarrow X-$ односторонняя обобщенная динамическая система.

ОПРЕДЕЛЕНИЕ 8. Точкой покоя односторонней динамической системы $\Psi$ назЫвается точка $x_{0} \in X$ такая, что $x_{0} \in \Psi\left(x_{0}, t\right)$ для любого $t \in \mathbb{R}_{+}$.

Наличие точки покоя динамической системы эквивалентно сушествованию постоянной траектории этой системы.

Справедливо следуюшее утверждение (см. [1]).

Лемма 3. Пусть $X$ - компактное метрическое пространство, М-отображение $F: X \times \mathbb{R}_{+} \rightarrow \mathrm{K}(X)$ удовлетворяет следующим условиям:

1) $F(x, 0)=x$ для любого $x \in X$;

2) $F\left(x, t_{1}+t_{2}\right) \supset F\left(F\left(x, t_{1}\right), t_{2}\right)$ для $0<t_{1}, t_{2}<\infty$;

3) $F$ - полунепрерывное сверху М-отобрахсение.

Тогда для существования точки покоя системы $F$ необходимо и достаточно, чтобы для любого $\varepsilon>0$ нашлись $x_{\varepsilon} \in X u \delta_{\varepsilon} \in(0, \varepsilon)$ такие, что $x_{\varepsilon} \in F\left(x_{\varepsilon}, \delta_{\varepsilon}\right)$.

Очевидно, что односторонняя динамическая система $\Psi$ удовлетворяет условиям этой леммы. Следовательно, лемма 3 позволяет сводить теоремы существования точек покоя односторонних обобщенных динамических систем к теоремам о неподвижных точках М-отображений.

ОПРЕДЕЛЕНИЕ 9. Метрическое пространство $Y$ называется абсолютным окрестностным ретрактом (ANR-пространством), если для любого гомеоморфизма $h$, отображающего $Y$ на замкнутое подмножество $h(Y)$ метрического пространства $X$, множество $h(Y)$ является ретрактом некоторого открытого множества $U \subset X$ такого, что $h(Y) \subset U$.

Справедлива следующая теорема (см., например, [4]).

Tеорема 2. Пусть $Y$ - компактное ацикличное ANR-пространство и М-отображсене $F: Y \rightarrow \mathrm{K}(Y)$ удовлетворяет следующему условию:

существуют метрическое пространство $Z$, непрерывное отобрахсене $l: Z \rightarrow Y$ и полунепрерывное сверху М-отображение $G: Y \rightarrow \mathrm{K}(Z)$ с аииклическими образами такие, что $F(x)=l \cdot G(x)$ для любого $x \in Y$.

Тогда М-отображсние $F$ имеет в $Y$ неподвижную точку.

Заметим, что эта теорема является обобщением теоремы Какутани (см. [11]), так как любое замкнутое вьпуклое ограниченное подмножество конечномерного пространства $\mathbb{R}^{n}$ является компактньм ацикличным ANR-пространством.

Пусть $\Psi: X \times \mathbb{R}_{+} \rightarrow X-$ односторонняя динамическая система.

ОПРЕДЕЛЕНИЕ 10. Множество $Y \subset X$ называется инвариантным для $\Psi$, если для любых $x \in Y, t \in \mathbb{R}_{+}$справедливо включение $\Psi(x, t) \subset Y$.

ТеОрема 3. Пусть $X$ - компактное метрическое пространство, $\Psi: X \times \mathbb{R}_{+} \rightarrow$ $\mathrm{K}(X)$ - односторонняя обобщенная динамическая система. Пусть компакт $Y \subset X$ является инвариантным множсеством для $\Psi$. Если

1) множсество $Y$ является аиикличным ANR-пространством,

2) динамическая система $\Psi$ является аппроксимируемой, 
то динамическая система $\Psi$ имеет в $Y$ точку покоя.

ДокАЗАТЕЛЬСТво. В силу теоремы 1 множество $\Sigma\left(x_{0},[0, T]\right)$ является ацикличным для любых $x_{0} \in X, T>0$. Рассмотрим М-отображение $F_{T}: Y \rightarrow \mathrm{K}(Y)$, определенное условием

$$
F_{T}\left(x_{0}\right)=\left\{y \in Y \mid y=x(T), x(\cdot) \in \Sigma\left(x_{0},[0, T]\right)\right\} .
$$

В силу свойства 3 траекторий обобщенных динамических систем очевидно, что $F_{T}\left(x_{0}\right)$ $=\Psi\left(x_{0}, T\right)$ для любых $x_{0} \in Y, T>0$. С другой стороны, $F_{T}\left(x_{0}\right)=l \cdot \Sigma\left(x_{0}\right)$, где $\Sigma: Y \rightarrow$ $\mathrm{K}\left(C_{([0, T], X)}\right), \Sigma\left(x_{0}\right)=\Sigma\left(x_{0},[0, T]\right)$ и имеет ациклические образы, a $l: C_{([0, T], X)} \rightarrow X$, $l(x(\cdot))=x(T)$.

В силу леммы 1 М-отображение $\Sigma$ является полунепрерывным сверху. Очевидно также, что отображение $l$ является непрерьвным. Поэтому М-отображение $F_{T}$ удовлетворяет условиям теоремы 2. Следовательно, у него существует неподвижная точка в $Y$.

Таким образом, в силу произвольности $T$ получаем, что для любого $\varepsilon>0$ существует точка $x_{\varepsilon} \in Y$ такая, что $x_{\varepsilon} \in F_{\varepsilon}\left(x_{\varepsilon}\right)=\Psi\left(x_{\varepsilon}, \varepsilon\right)$. Следовательно, в силу леммы 3 односторонняя динамическая система $\Psi$ имеет точку покоя в $Y$.

Теорема доказана.

Используя число Лефшеца для многозначных отображений (см. [4]) и ацикличность М-отображения $\Sigma$, можно доказать следующее утверждение.

Tеорема 4. Пусть $X$ - компактное ANR-пространство, $\Psi: X \times \mathbb{R}_{+} \rightarrow \mathrm{K}(X)$ односторонняя динамическая система. Пусть выполнены следующие условия:

1) динамическая система $\Psi$ является аппроксимируемой на $X$;

2) существует $T \in \mathbb{R}_{+}$такое, что мнохсество $\Psi(X, T)$ ациклично в $X$.

Тогда система $\Psi$ имеет в $X$ точку покоя.

ДокАЗАтЕльство. Пусть $t_{n}=T / n$. Рассмотрим М-отображение $F_{n}=F_{t_{n}}: X \rightarrow$ $\mathrm{K}(X)$, определенное условием

$$
F_{n}\left(x_{0}\right)=\left\{y \in Y \mid y=x\left(t_{n}\right), x(\cdot) \in \Sigma\left(x_{0},\left[0, t_{n}\right]\right)\right\}
$$

В силу свойств обобщенной динамической системы имеем равенство $F_{T}(x)=\left(F_{n}\right)^{n}(x)$. Действительно, если $y \in F_{T}\left(x_{0}\right)$, то сушествует траектория $x(\cdot) \in \Sigma\left(x_{0},[0, T]\right)$ такая, что $x(T)=y$. Имеем,

$$
x\left(t_{n}\right) \in F_{n}\left(x_{0}\right), \quad x\left(2 t_{n}\right) \in \Psi\left(x\left(t_{n}\right), t_{n}\right)=F_{n}\left(x\left(t_{n}\right)\right) \in\left(F_{n}\right)^{2}\left(x_{0}\right) .
$$

Продолжая эту процедуру дальше, получим $y=x(T) \in\left(F_{n}\right)^{n}(x)$.

В обратную сторону, пусть $y \in\left(F_{n}\right)^{n}(x)$. Тогда найдется точка $y_{n-1} \in\left(F_{n}\right)^{n-1}(x)$ такая, что $y \in F_{n}\left(y_{n-1}\right)$. Следовательно, сушествует траектория $x_{n-1}(\cdot) \in \Sigma\left(y_{n-1}\right.$, $\left.\left[0, t_{n}\right]\right)$. Тогда отображение $z_{n-1}(t)=x_{n-1}\left(t-(n-1) t_{n}\right)$ будет траекторией динамической системы на промежутке $\left[(n-1) t_{n}, T\right]$. Аналогичньй процесс построения траекторий можно продолжить дальше, тогда получим последовательность траекторий $z_{0}(\cdot)$, $z_{1}(\cdot), \ldots, z_{n-1}(\cdot)$ на соответствуюших промежутках $\left[0, t_{n}\right],\left[t_{n}, 2 t_{n}\right], \ldots,\left[(n-1) t_{n}, T\right]$. 
Склеив эти траектории, получим траекторию $z(\cdot)$ такую, что $y=z(T)$. Это и доказывает требуемое равенство.

Как и в теореме 3, М-отображение $F_{n}(x)=l \cdot \Sigma(x)$, где $\Sigma: X \rightarrow \mathrm{K}\left(C_{\left(\left[0, t_{n}\right], X\right)}\right)$, $\Sigma(x)=\Sigma\left(x,\left[0, t_{n}\right]\right)$ и имеет ациклические образы, а $l: C_{\left(\left[0, t_{n}\right], X\right)} \rightarrow X, l(x(\cdot))=x\left(t_{n}\right)$.

Для таких М-отображений построено число Лефшеца и доказана теорема о неподвижной точке (см., например, [4]). Можно доказать (см. [4]), что число Лефшеца $\Lambda\left(F_{n}\right)=1$, так как $\left(F_{n}\right)^{n}(X)$ является ацикличным множеством в $X$. Следовательно, М-отображение $F_{n}$ имеет в $X$ неподвижную точку $x_{n}$, т.е. $x_{n} \in F_{n}\left(x_{n}\right)=\Psi\left(t_{n}, x_{n}\right)$.

Так как такую точку $x_{n}$ можно построить для любого $n$, получаем последовательность точек $\left\{x_{n}\right\}$, которые являются неподвижными для М-отображения $\Psi$ при $t_{n}=T / n$. В силу того, что последовательность $\left\{t_{n}\right\}$ стремится к 0 , вьполнены все условия леммы 3 . Следовательно, динамическая система $\Psi$ имеет точку покоя, что и доказьвает теорему.

\section{СПИСОК ЦИТИРОВАННОЙ ЛИТЕРАТУРЫ}

[1] Мышкис А. Д. Обобщения теоремы о точке покоя динамической системы внутри замкнутой траектории // Матем. сб. 1954. Т. 34(76). № 3. С. 525-540.

[2] Борисович Ю. Г., Гельман Б. Д., Мышкис А. Д., Обуховский В. В. Топологические методы в теории неподвижных точек многозначных точек многозначных отображений // УМН. 1980. Т. 35. № 1. С. 59-126.

[3] Обуховский В. В. Асимптотические теоремы о неподвижной точке и точке покоя динамических систем без единственности // Сб. работ аспирантов по теории функций и дифференц. уравнениям. Воронеж: ВГУ, 1974. С. 30-38.

[4] Gorniewicz L. Homological methods in fixed point theory of multi-valued maps. Dissertationes Math. (Rozprawy Mat.). V. 129. Warsaw: Polish Acad. Sci., 1975.

[5] Борисович Ю. Г., Гельман Б. Д., Мышкис А. Д., Обуховский В. В. Введение в теорию многозначных отображений. Воронеж: Изд-во ВГУ, 1986.

[6] Сибирский К. С., Шубэ А. С. Полудинамические системы. Кишинев: Штиинца, 1987.

[7] Szego G. P., Treccani G. Semigruppi di transformazioni multivoche. Lecture Notes in Math. V. 101. Berlin: Springer, 1969.

[8] Барбашин Е. А. К теории обобщенных динамических систем // Уч. записки МГУ. 1948. T. 135. № 2. C. 110-113.

[9] Спеньер Э. Алгебраическая топология. М.: Мир, 1971.

[10] Скляренко Е.Г. О некоторых приложениях теории пучков в общей топологии // УМН. 1964. T. 19. № 6. C. 47-70.

[11] Kakutani S. A generalization of Brouwer's fixed point theorem // Duke Math. J. 1941. V. 8. № 3. P. 457-459. 